\title{
DNA evolution depends on differential methylation patterns in rat speciation
}

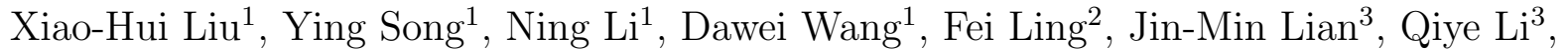 \\ Yabin $\mathrm{Jin}^{2}$, Zhiyong Feng ${ }^{4}$, Lin Cong ${ }^{5}$, Dandan $\mathrm{Yao}^{4}$, Chan $\mathrm{Luo}^{6}$, and Ming D. $\mathrm{Li}^{7}$ \\ ${ }^{1}$ Chinese Academy of Agricultural Sciences Institute of Plant Protection \\ ${ }^{2}$ South China University of Technology School of Bioscience and Bioengineering \\ ${ }^{3}$ China National GeneBank \\ ${ }^{4}$ Guangdong Academy of Agricultural Sciences \\ ${ }^{5}$ Plant Protection Institute, Heilongjiang Academy of Agricultural Sciences \\ ${ }^{6}$ Heilongjiang Academy of Agricultural Sciences \\ ${ }^{7}$ Zhejiang University School of Medicine First Affiliated Hospital
}

June 1, 2021

\begin{abstract}
The fixation of phenotypes and underlying alleles is a typical evolutionary process in speciation. As the primary molecular basis of phenotypic plasticity, epigenetic mechanisms also play an essential role in maintaining phenotypes. However, whether and how DNA evolution was shaped by epigenetic alteration remains unknown, especially accompanied DNA fixation in speciation. We used sperm methylomes of three rat subspecies as epigenetic markers and screened out genomic regions that experienced distinct differential methylation. To obtain independent results, they were further filtrated according to genomic locations to guarantee that their evolutionary features were not interactively affected by nearby DMRs (differentially methylated regions) of other datasets. By analyzing intraspecies and interspecies phylogenetic relationships, we showed that, in the same genomic regions, the significantly accelerated DNA evolution only occurred in individuals or lineages that experienced differential methylation. Across the same genomes, differential methylation led to a significant increase of $F_{S T}$ only in lineage-specific DMRs and a significant increase of $\pi$ in both individual-specific and lineage-specific DMRs. Correlations among methylation, $\pi$ and $F_{S T}$ showed that it was methylation consistency rather than the absolute methylation difference that significantly influenced both $\pi$ and $F_{S T}$. The change of both $\pi$ and DNA fixation depended on the degree of intraspecies methylation consistency. While the breakdown of methylation consistency facilitated the promotion of $\pi$, the maintenance of methylation consistency facilitated the acceleration of DNA fixation.
\end{abstract}

\section{Hosted file}

MS_20210514.pdf available at https ://authorea.com/users/417310/articles/524459-dna-evolutiondepends-on-differential-methylation-patterns-in-rat-speciation 